\title{
Robustness of Time Reversal for Waves in Time-dependent Random Media
}

\author{
Daniel G. Alfaro Vigo \\ Instituto de Matemática Pura e Aplicada, Est. D Castorina 110 , Jardim \\ Botânico, Rio de Janeiro, RJ 22460-320, Brazil. \\ Jean-Pierre Fouque \\ Department of Mathematics, North Carolina State University, Raleigh NC \\ 27695-8205. \\ Josselin Garnier ${ }^{1}$ \\ Laboratoire de Statistique et Probabilités, Université Paul Sabatier, 118 Route de \\ Narbonne, 31062 Toulouse Cedex 4, France. Tel: (33).5.61.55.62.20, Fax: \\ (33).5.61.55.60.89. \\ André Nachbin \\ Instituto de Matemática Pura e Aplicada, Est. D Castorina 110 , Jardim \\ Botânico, Rio de Janeiro, RJ 22460-320, Brazil.
}

\begin{abstract}
This paper addresses the impact of time fluctuations of a random medium on refocusing during a time-reversal experiment. Even in the presence of moderate timeperturbations a coherent refocused pulse is observed. The theory predicts the level of recompression observed as well as the conditions for the loss of statistical stabilization. It is shown that the statistical properties of the refocused pulse depend on a simple set of parameters that describe the correlation degree of the medium. The refocused pulse has in general a random shape that can be described in terms of a system of stochastic transport equations driven by a single Brownian motion. Pulse stabilization is also demonstrated for some particular configurations, and the convolution kernel that describes the pulse reshaping is explicitly computed. Numerical simulations are presented and show a very good agreement with the theoretical predictions, thus providing a clear illustration of the robustness of time reversal.
\end{abstract}

Key words: Inhomogeneous media, asymptotic theory, time reversal.

$A M S: 35 \mathrm{~L} 05,60 \mathrm{H} 15,74 \mathrm{~J} 20$. 


\section{Introduction}

In this paper we analyze the effect of a time dependent random medium on the time-reversed refocusing phenomenon. We consider linear acoustic waves propagating in a disordered one-dimensional medium. We demonstrate that even in the presence of moderate time-perturbations a coherent refocused pulse is observed. The theory also predicts the level of recompression observed as well as the conditions for the loss of statistical stabilization.

Time-reversal refocusing for waves propagating in inhomogeneous media have been recently observed and studied experimentally in various contexts, e.g. ultrasound, underwater acoustics, see for instance the review [13]. Important potential applications have been proposed in various fields, for instance imaging $[22,14]$ and communication [12]. A time-reversal mirror is, roughly speaking, a device which is capable of receiving a signal in time, keeping it in memory and sending it back into the medium in the reversed direction of time. The main effect is the refocusing of the scattered signal after time-reversal in a random medium. Surprisingly, the refocused pulse shape only depends on the statistical properties of the random medium, and not on the particular realization of the medium. The full mathematical understanding, meaning both modeling of the physical problem and derivation of the time-reversal effect, is a complex problem. The study of the one-dimensional linear case is now well understood $[11,23,15]$ as well as the three-dimensional waves in the parabolic or paraxial regime $[4,8,21]$. In this paper we consider the situation where the medium changes from the forward experiment to the time-reversed experiment. This characterizes a time-dependent medium. Experimental results on the robustness of acoustic time-reversal refocusing in a multiple scattering medium undergoing perturbations are presented in [25]. Here we address theoretically and numerically for the first time the impact of such a time-fluctuating medium on the statistical properties of the pulse refocusing.

The paper is organized as follows. In Sections 2-6 we derive integral representations of the refocused pulse shapes in terms of reflection and transmission coefficients for the wave modes. Section 7, resp. 8, is devoted to the thorough analysis of time-reversal experiments in reflection, resp. in transmission. Section 9 presents results from full numerical simulations.

Email addresses: dgalfaro@impa.br (Daniel G. Alfaro Vigo), fouque@math.ncsu.edu (Jean-Pierre Fouque), garnier@cict.fr (Josselin Garnier), nachbin@impa.br (André Nachbin).

1 Corresponding author 


\section{The acoustic model}

We consider the acoustic wave equation

$$
\frac{1}{K} \frac{\partial p}{\partial t}+\frac{\partial u}{\partial z}=0, \quad \rho \frac{\partial u}{\partial t}+\frac{\partial p}{\partial z}=0
$$

where $p$ is the pressure and $u$ is the velocity. $z$ and $t$ are the space and time coordinates, respectively. $\rho$ is the density of the medium and $K$ the bulk modulus. The medium is described by

$$
\begin{gathered}
\rho= \begin{cases}1+\nu\left(\frac{z}{\varepsilon^{2}}\right) & \text { if }-L<z<0 \\
1 & \text { if } z>0 \text { or } z<-L\end{cases} \\
\frac{1}{K}= \begin{cases}1+\eta\left(\frac{z}{\varepsilon^{2}}\right) & \text { if }-L<z<0 \\
1 & \text { if } z>0 \text { or } z<-L\end{cases}
\end{gathered}
$$

where $(\eta, \nu)$ is a $\mathbb{R}^{2}$-valued mixing process. The dimensionless small parameter $\varepsilon^{2}$ characterizes the ratio of the correlation radius of the fluctuations of the medium and the typical size of the medium. These fluctuations are modeled by the zero-mean stationary random process $(\eta, \nu)(z)$. The processes $\eta$ and $\nu$ are assumed to be bounded by a deterministic constant less than 1 and to have strong ergodic properties. We may think for instance that $(\eta, \nu)$ is a function of a Markov, stationary, ergodic process on a compact space satisfying the Fredholm alternative [20]. We can also work with the class of the $\phi$-mixing processes with $\phi \in L^{1 / 2}[16]$.

We consider the problem on the finite slab $-L \leq z \leq 0$ where boundary conditions will be imposed at $-L$ and 0 corresponding to a pulse entering the slab from the right at $z=0$. The quantities of interest, the transmitted and reflected waves, will be observed in time at the extremities $z=-L$ and $z=0$, respectively.

We consider a pulse whose typical wavelength is of order $\varepsilon$, that is to say small compared to the size of the medium, but large with respect to the correlation length of the random medium. Accordingly, the pressure field of the incoming pulse shape will be taken of the form $f\left(\frac{t}{\varepsilon}\right)$. 


\section{Propagator formulation}

In this section we first express the scattering problem as a two point boundary value problem in the frequency domain, and then rewrite it as an initial value problem in terms of the propagator. This study follows the lines of the analysis carried out in Ref. [3]. We consider the random acoustic equation (1) and take the scaled time Fourier transform

$\hat{u}^{\varepsilon}(\omega, z)=\frac{1}{2 \pi \varepsilon} \int u(t, z) \exp \left(-\frac{i \omega t}{\varepsilon}\right) d t, \quad \hat{p}^{\varepsilon}(\omega, z)=\frac{1}{2 \pi \varepsilon} \int p(t, z) \exp \left(-\frac{i \omega t}{\varepsilon}\right) d t$

so that the system reduces to a set of ordinary differential equations:

$$
\frac{\partial \hat{p}^{\varepsilon}}{\partial z}+\frac{i \omega}{\varepsilon}\left(1+\nu\left(\frac{z}{\varepsilon^{2}}\right)\right) \hat{u}^{\varepsilon}=0, \quad \frac{\partial \hat{u}^{\varepsilon}}{\partial z}+\frac{i \omega}{\varepsilon}\left(1+\eta\left(\frac{z}{\varepsilon^{2}}\right)\right) \hat{p}^{\varepsilon}=0
$$

We can decompose the wave into right-going modes $A^{\varepsilon}$ and left-going modes $B^{\varepsilon}$

$$
A^{\varepsilon}(\omega, z)=\frac{\hat{p}^{\varepsilon}(\omega, z)+\hat{u}^{\varepsilon}(\omega, z)}{2}, \quad B^{\varepsilon}(\omega, z)=\frac{\hat{p}^{\varepsilon}(\omega, z)-\hat{u}^{\varepsilon}(\omega, z)}{2}
$$

and we look at the waves along the frequency-dependent characteristics defined by

$$
a^{\varepsilon}(\omega, z)=A^{\varepsilon}(\omega, z) \exp \left(\frac{i \omega z}{\varepsilon}\right), \quad b^{\varepsilon}(\omega, z)=B^{\varepsilon}(\omega, z) \exp \left(-\frac{i \omega z}{\varepsilon}\right) .
$$

$\left(a^{\varepsilon}, b^{\varepsilon}\right)$ satisfy the linear equation

$$
\frac{\partial}{\partial z}\left(\begin{array}{l}
a^{\varepsilon} \\
b^{\varepsilon}
\end{array}\right)(\omega, z)=Q^{\varepsilon}(\omega, z)\left(\begin{array}{l}
a^{\varepsilon} \\
b^{\varepsilon}
\end{array}\right)(\omega, z) .
$$

where the complex $2 \times 2$ matrix $Q^{\varepsilon}$ is given by

$$
Q^{\varepsilon}(\omega, z)=\frac{i \omega}{2}\left(\begin{array}{cc}
-m^{\varepsilon}(z) & -n^{\varepsilon}(z) e^{\frac{2 i \omega z}{\varepsilon}} \\
n^{\varepsilon}(z) e^{-\frac{2 i \omega z}{\varepsilon}} & m^{\varepsilon}(z)
\end{array}\right)
$$

with

$$
\begin{aligned}
& m(z)=\eta(z)+\nu(z), \quad n(z)=\eta(z)-\nu(z) \\
& m^{\varepsilon}(z)=\frac{1}{\varepsilon} m\left(\frac{z}{\varepsilon^{2}}\right), \quad n^{\varepsilon}(z)=\frac{1}{\varepsilon} n\left(\frac{z}{\varepsilon^{2}}\right)
\end{aligned}
$$




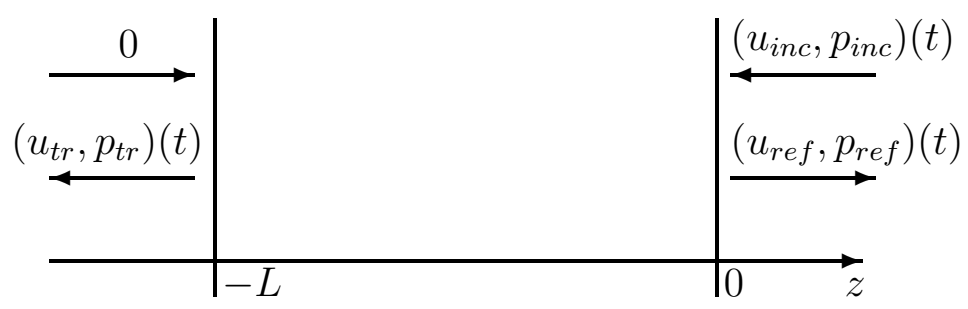

Fig. 1. Scattering problem.

We assume that a left-going pulse is incoming from the right and is scattered into a reflected wave at $z=0$ and a transmitted wave at $z=-L$ (see Figure 1 ). The incoming pulse shape is given by the pressure function $f\left(\frac{t}{\varepsilon}\right)$ where $f$ is assumed to be a $L^{1}$ function compactly supported in the Fourier domain

$$
p_{i n c}(t, z=0)=\int \hat{f}(\omega) \exp \left(\frac{i \omega t}{\varepsilon}\right) d \omega
$$

and $u_{\text {inc }}(t, z=0)=-p_{\text {inc }}(t, z=0)$. We also impose a radiation condition at $-L$ corresponding to the absence of right-going wave at the left hand-side of the slab $[-L, 0]$. The two-point boundary value problem consisting of the system $(7)$ for $z \in[0, L]$ together with the boundary conditions $b^{\varepsilon}(\omega, z=0)=$ $\hat{f}(\omega), a^{\varepsilon}(\omega, z=-L)=0$ is then well-posed.

It is convenient to transform the two-point boundary value problem into an initial value problem by introducing the propagator $Y^{\varepsilon}(\omega,-L, z)$ which is a complex $2 \times 2$ matrix solution of

$$
\frac{\partial Y^{\varepsilon}}{\partial z}(\omega,-L, z)=Q^{\varepsilon}(\omega, z) Y^{\varepsilon}(\omega,-L, z), \quad Y^{\varepsilon}(\omega,-L, z=-L)=I d_{\mathbb{C}^{2}}
$$

such that

$$
Y^{\varepsilon}(\omega,-L, z)\left(\begin{array}{c}
a^{\varepsilon}(\omega,-L) \\
b^{\varepsilon}(\omega,-L)
\end{array}\right)=\left(\begin{array}{l}
a^{\varepsilon}(\omega, z) \\
b^{\varepsilon}(\omega, z)
\end{array}\right) .
$$

By the form (8) of the matrix $Q^{\varepsilon}$, if the column vector $\left(a_{1}^{\varepsilon}, b_{1}^{\varepsilon}\right)^{T}$ is solution of equation (7) with the initial conditions

$$
a_{1}^{\varepsilon}(\omega,-L)=1, \quad b_{1}^{\varepsilon}(\omega,-L)=0,
$$

then the column vector $\left(\overline{b_{1}^{\varepsilon}}, \overline{a_{1}^{\varepsilon}}\right)^{T}$ is another solution linearly independent of the first solution, so that the propagator matrix $Y^{\varepsilon}$ can be written as:

$$
Y^{\varepsilon}(\omega,-L, z)=\left(\begin{array}{l}
a_{1}^{\varepsilon} \overline{b_{1}^{\varepsilon}} \\
b_{1}^{\varepsilon} \overline{a_{1}^{\varepsilon}}
\end{array}\right)(\omega, z) .
$$




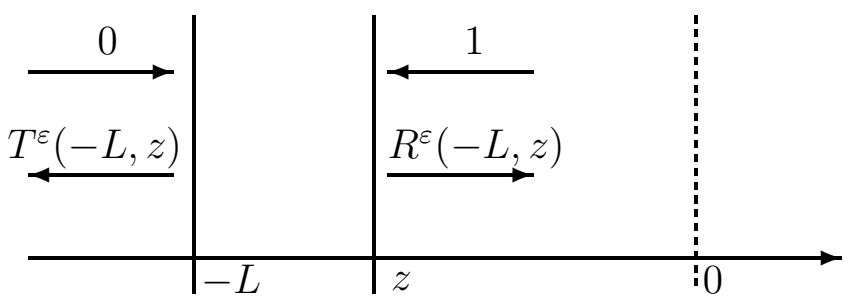

Fig. 2. Reflection and transmission coefficients.

Note also that the matrix $Q^{\varepsilon}$ has zero trace, so that the determinant of $Y^{\varepsilon}$ is conserved and $\left(a_{1}^{\varepsilon}, b_{1}^{\varepsilon}\right)$ satisfies the relation:

$$
\operatorname{det} Y^{\varepsilon}=\left|a_{1}^{\varepsilon}\right|^{2}-\left|b_{1}^{\varepsilon}\right|^{2}=1 \text {. }
$$

We can now define the transmission and reflection coefficients $T^{\varepsilon}(\omega,-L, z)$ and $R^{\varepsilon}(\omega,-L, z)$, respectively, for a slab $[-L, z]$ by (see also Figure 2 ):

$$
Y^{\varepsilon}(\omega,-L, z)\left(\begin{array}{c}
0 \\
T^{\varepsilon}(\omega,-L, z)
\end{array}\right)=\left(\begin{array}{c}
R^{\varepsilon}(\omega,-L, z) \\
1
\end{array}\right) .
$$

In terms of the propagator entries they are given by

$$
R^{\varepsilon}(\omega,-L, z)=\frac{\overline{b_{1}^{\varepsilon}}}{\overline{a_{1}^{\varepsilon}}}(\omega, z), \quad T^{\varepsilon}(\omega,-L, z)=\frac{1}{\overline{a_{1}^{\varepsilon}}}(\omega, z)
$$

and they satisfy the closed form nonlinear differential system

$$
\begin{aligned}
& \frac{\partial R^{\varepsilon}}{\partial z}=-i \omega m^{\varepsilon}(z) R^{\varepsilon}-\frac{i \omega}{2} e^{-\frac{2 i \omega z}{\varepsilon}} n^{\varepsilon}(z)\left(R^{\varepsilon}\right)^{2}-\frac{i \omega}{2} e^{\frac{2 i \omega z}{\varepsilon}} n^{\varepsilon}(z), \\
& \frac{\partial T^{\varepsilon}}{\partial z}=-\frac{i \omega}{2} T^{\varepsilon}\left(e^{-\frac{2 i \omega z}{\varepsilon}} n^{\varepsilon}(z) R^{\varepsilon}+m^{\varepsilon}(z)\right),
\end{aligned}
$$

with the initial conditions at $z=-L$

$$
R^{\varepsilon}(\omega,-L, z=-L)=0, \quad T^{\varepsilon}(\omega,-L, z=-L)=1 .
$$

Note that Eq. (13) implies the conservation of energy relation

$$
\left|R^{\varepsilon}\right|^{2}+\left|T^{\varepsilon}\right|^{2}=1
$$

and in turn the uniform boundedness of the transmission and reflection coefficients. Note also that $R^{\varepsilon}$ and $T^{\varepsilon}$ are the reflection and transmission coefficients for the modified characteristics (6). In terms of the real characteristics the reflection and transmission coefficients are $R^{\varepsilon}$ and $T^{\varepsilon} \exp (-i \omega L / \varepsilon)$, respectively. 
The transmitted wave at time $t$, denoted by $\left(u_{t r}, p_{t r}\right)$, is the left-going wave which admits the following integral representation in terms of the transmission coefficients

$$
p_{t r}(t, z=-L)=\int \hat{f}(\omega) T^{\varepsilon}(\omega,-L, 0) \exp \left(\frac{i \omega(t-L)}{\varepsilon}\right) d \omega
$$

and $u_{t r}(t, z=-L)=-p_{t r}(t, z=-L)$. The reflected wave at time $t$, denoted by $\left(u_{r e f}, p_{r e f}\right)$, is the right-going wave which admits the following integral representation in terms of the reflection coefficients

$$
p_{\text {ref }}(t, z=0)=\int \hat{f}(\omega) R^{\varepsilon}(\omega,-L, 0) \exp \left(\frac{i \omega t}{\varepsilon}\right) d \omega
$$

and $u_{r e f}(t, z=-L)=p_{r e f}(t, z=-L)$.

\section{Transmitted front wave}

Before going into time reversal we give an integral representation for the $c o$ herent transmitted wave front observed at $z=-L$ around the expected arrival time L. By Eq. (17), the transmitted front observed in the time scale of the initial pulse is given by:

$$
p_{t r}(L+\varepsilon t, z=-L)=\int e^{i \omega t} \hat{f}(\omega) T^{\varepsilon}(\omega,-L, 0) d \omega .
$$

The transmission coefficients are given by $T^{\varepsilon}(\omega,-L, 0)=1 / \overline{a_{1}^{\varepsilon}}(\omega, 0)$ where $a_{1}^{\varepsilon}$ satisfies (7) with the initial conditions (12). The asymptotic behavior of $p_{t r}$ as $\varepsilon \rightarrow 0$ has been studied in $[10,17,24]$. It is proved that the process $\left(p_{t r}(L+\right.$ $\varepsilon t, z=-L))_{t \in(-\infty,+\infty)}$ converges in the space of the continuous functions to

$$
P_{t r}(t)=\int \hat{f}(\omega) \exp \left(i \omega\left(t-\frac{\sqrt{\alpha_{m}}}{\sqrt{2}} B_{L}\right)-\frac{\alpha_{n} \omega^{2}}{4} L\right) d \omega
$$

where $B_{L}$ is a standard Brownian motion and

$$
\alpha_{m}=\int_{0}^{\infty} \mathbb{E}[m(0) m(z)] d z, \quad \alpha_{n}=\int_{0}^{\infty} \mathbb{E}[n(0) n(z)] d z .
$$

Using convolution operators the transmitted front can be written as

$$
P_{t r}(t)=f * K\left(t-\frac{\sqrt{\alpha_{m}}}{\sqrt{2}} B_{L}\right)
$$


which means that a random Gaussian centering appears through the Brownian motion $B_{L}$ while the pulse shape spreads in a deterministic way through the convolution by the Gaussian kernel $K$ whose Fourier transform is

$$
\hat{K}(\omega)=\exp \left(-\alpha_{n} \omega^{2} L / 4\right)
$$

Note that the parameter $\alpha_{m}$ imposes the random time shift, while the parameter $\alpha_{n}$ imposes the deterministic reshaping of the pulse. This result corresponds to the O'Doherty-Anstey (ODA) theory celebrated in geophysics [18].

\section{Time reversal in reflection (TRR)}

The first step of the time reversal procedure consists in recording the reflected signal at $z=0$ up to a certain time. During this first part of the experiment the fluctuations of the medium are represented by the processes $\eta_{i}, \nu_{i}$, and the sum and difference by $m_{i}$ and $n_{i}$, respectively. It turns out that as $\varepsilon \rightarrow 0$ the interesting asymptotic regime arises when we record the signal up to a large time of order 1 which we denote by $t_{1}$ with $t_{1}>0$. In the next step of the time reversal procedure a piece of the recorded signal is cut using a cut-off function $s \mapsto G_{t_{1}}(s)$ where the support of $G_{t_{1}}$ is included in $\left[0, t_{1}\right]$ :

$$
p_{\text {ref,cut }}(t)=p_{\text {ref }}(t) G_{t_{1}}(t) .
$$

One then time reverses that piece of signal and sends it back into the medium. Observe that the part of the wave which came in last will go out first. We also change the sign of the recorded velocity so as to generate a pure left-going wave. The time-reversed pressure wave sent back into the medium is given by

$$
\begin{aligned}
p_{\text {inc }(T R R)}(t, z=0) & =p_{\text {ref,cut }}\left(t_{1}-t\right) \\
& =\frac{1}{\varepsilon} \iint \exp \left(\frac{i \omega\left(t_{1}-t\right)}{\varepsilon}\right) \hat{p}_{r e f}^{\varepsilon}\left(\omega^{\prime}\right) \hat{G}_{t_{1}}\left(\frac{\omega-\omega^{\prime}}{\varepsilon}\right) d \omega^{\prime} d \omega
\end{aligned}
$$

where $\hat{G}_{t_{1}}$ is the Fourier transform of $G_{t_{1}}$. Using the fact that $p_{i n c(T R R)}$ is real valued we get

$$
p_{\text {inc(TRR) }}(t, z=0)=\frac{1}{\varepsilon} \iint \exp \left(\frac{i \omega\left(t-t_{1}\right)}{\varepsilon}\right){\overline{\hat{p}_{r e f}}}_{r}\left(\omega^{\prime}\right) \overline{\hat{G}}_{t_{1}}\left(\frac{\omega-\omega^{\prime}}{\varepsilon}\right) d \omega^{\prime} d \omega,
$$

where TRR stands for "Time Reversal in Reflection". The fluctuations of the medium may have been modified between the time windows corresponding to the first and second parts of the time-reversal experiment. We shall denote by $\left(\eta_{i i}, \nu_{i i}\right)$ the random process that represents the fluctuations of the medium 
during the second part of the time-reversal experiment. We also denote by $m_{i i}$ and $n_{i i}$ their sum and difference. $\left(\eta_{i i}, \nu_{i i}\right)$ is assumed to have the same statistical distribution as $\left(\eta_{i}, \nu_{i}\right)$, but they are different realizations and we shall see that the impact of this difference will be characterized by a correlation degree between the processes $\left(\eta_{i}, \nu_{i}\right)$ and $\left(\eta_{i i}, \nu_{i i}\right)$.

This new incoming signal re-propagates into the medium $\left(\eta_{i i}, \nu_{i i}\right)$, and generates a new reflected signal which we observe at the time $t_{2}+\varepsilon t$, that is, around the time $t_{2}$ in the scale of the initial pulse. In terms of the reflection coefficients $R_{i i}^{\varepsilon}$ associated with the medium $\left(\eta_{i i}, \nu_{i i}\right)$, the observed reflected signal is given by

$$
p_{r e f(T R R)}\left(t_{2}+\varepsilon t, z=0\right)=\int \hat{p}_{i n c(T R)}^{\varepsilon}(\omega) R_{i i}^{\varepsilon}(\omega,-L, 0) e^{\frac{i \omega t_{2}}{\varepsilon}+i \omega t} d \omega .
$$

Substituting the expression of $\hat{p}_{i n c(T R R)}^{\varepsilon}$ into this equation yields the following representation of the reflected signal:

$$
\begin{aligned}
p_{r e f(T R R)}\left(t_{2}+\varepsilon t, z=0\right)=\frac{1}{\varepsilon} \iint & e^{i \omega t} e^{\frac{i \omega\left(t_{2}-t_{1}\right)}{\varepsilon}} \overline{\hat{f}}\left(\omega^{\prime}\right) \overline{\hat{G}}_{t_{1}}\left(\frac{\omega-\omega^{\prime}}{\varepsilon}\right) \\
& \times R_{i i}^{\varepsilon}(\omega,-L, 0) \overline{R_{i}^{\varepsilon}}\left(\omega^{\prime},-L, 0\right) d \omega^{\prime} d \omega .
\end{aligned}
$$

After the change of variable $\omega^{\prime}=\omega-\varepsilon h$ the representation becomes

$$
\begin{aligned}
p_{r e f(T R R)}\left(t_{2}+\varepsilon t, z=0\right)= & \iint e^{i \omega t} e^{\frac{i \omega\left(t_{2}-t_{1}\right)}{\varepsilon}} \overline{\hat{f}}(\omega-\varepsilon h) \overline{\hat{G}}_{t_{1}}(h) \\
& \times R_{i i}^{\varepsilon}(\omega,-L, 0) \overline{R_{i}^{\varepsilon}}(\omega-\varepsilon h,-L, 0) d h d \omega .
\end{aligned}
$$

Note that by Eq. (16) the reflection coefficients are bounded and we shall show that the rapid phase $\exp \left(i \omega\left(t_{2}-t_{1}\right) / \varepsilon\right)$ averages out the integral except when $t_{2}=t_{1}$. This means that refocusing can be observed only at the time $t_{2}=t_{1}$. The precise description of the refocused pulse will be carried out in Section 7.4 .

\section{Time reversal in transmission (TRT)}

The time reversal procedure consists in recording the transmitted signal at $z=-L$ over the time interval $\left[L+t_{0}, L+t_{1}\right]$. During this first part of the experiment the fluctuations of the medium is represented by the process $m_{i}$. A piece of the recorded signal is cut using a cut-off function $s \mapsto G_{t_{0}, t_{1}}(s-L)$ where the support of $G_{t_{0}, t_{1}}$ is included in $\left[t_{0}, t_{1}\right]$ :

$$
p_{t r, c u t}(t)=p_{t r}^{\varepsilon}(L+t, z=-L) G_{t_{0}, t_{1}}(t) .
$$




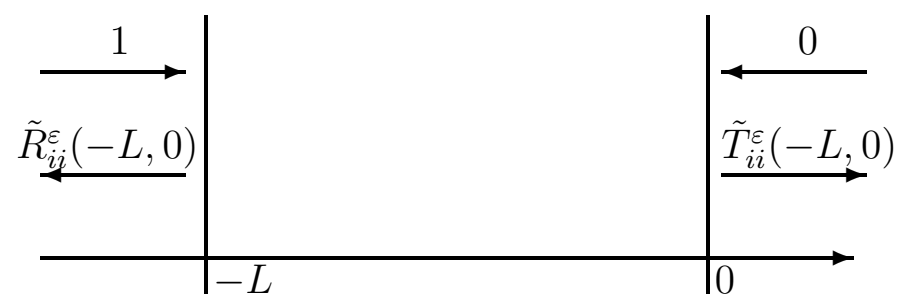

Fig. 3. Adjoint reflection and transmission coefficients for time reversal.

One then time reverses that piece of signal and sends it back into the medium by changing the sign of the velocity, so that we generate a right-going wave. The pressure sent back is $p_{t r, c u t}\left(t_{1}-t\right)$ which reads

$$
p_{\text {inc }(T R T)}(t, z=-L)==\frac{1}{\varepsilon} \iint \exp \left(\frac{i \omega\left(t_{1}-t\right)}{\varepsilon}\right) \hat{p}_{t r}^{\varepsilon}\left(\omega^{\prime}\right) \hat{G}_{t_{0}, t_{1}}\left(\frac{\omega-\omega^{\prime}}{\varepsilon}\right) d \omega^{\prime} d \omega
$$

where $\hat{p}_{t r}^{\varepsilon}$ is the scaled Fourier transform of the shifted received signal $t \mapsto$ $p_{t r}(L+t, z=-L)$,

$$
\hat{p}_{t r}^{\varepsilon}(\omega)=\hat{f}(\omega) T_{i}^{\varepsilon}(\omega,-L, 0),
$$

and $T_{i}^{\varepsilon}$ is the transmission coefficient associated with the fluctuations $\left(\eta_{i}, \nu_{i}\right)$. Since it is real-valued $p_{i n c(T R T)}$ also reads as:

$$
p_{\text {inc }(T R T)}(t, z=-L)=\frac{1}{\varepsilon} \iint \exp \left(\frac{i \omega\left(t-t_{1}\right)}{\varepsilon}\right) \overline{\hat{p}}_{t r}^{\varepsilon}\left(\omega^{\prime}\right) \overline{\hat{G}}_{t_{0}, t_{1}}\left(\frac{\omega-\omega^{\prime}}{\varepsilon}\right) d \omega^{\prime} d \omega .
$$

The fluctuations of the medium may have been modified between the time windows corresponding to the first and second parts of the time-reversal experiment. We shall denote by $\left(\eta_{i i}, \nu_{i i}\right)$ the random process that represents the fluctuations of the medium during the second part of the time-reversal experiment. We also denote by $\tilde{R}_{i i}^{\varepsilon}$ and $\tilde{T}_{i i}^{\varepsilon}$ the reflection and transmission coefficients for the experiment corresponding to a right-going input wave incoming from the left (see Figure 3). Using the propagator $Y_{i i}^{\varepsilon}$ defined as in Eq. (11) with the process $\left(\eta_{i i}, \nu_{i i}\right), \tilde{R}_{i i}^{\varepsilon}$ and $\tilde{T}_{i i}^{\varepsilon}$ obey the relation

$$
Y_{i i}^{\varepsilon}(\omega,-L, 0)\left(\begin{array}{c}
1 \\
\tilde{R}_{i i}^{\varepsilon}(\omega,-L, 0)
\end{array}\right)=\left(\begin{array}{c}
\tilde{T}_{i i}^{\varepsilon}(\omega,-L, 0) \\
0
\end{array}\right) .
$$

In terms of the propagator entries they are given by

$$
\tilde{R}_{i i}^{\varepsilon}(\omega,-L, 0)=-\frac{b_{1, i i}^{\varepsilon}}{\overline{a_{1, i i}^{\varepsilon}}}(\omega, 0), \quad \tilde{T}_{i i}^{\varepsilon}(\omega,-L, 0)=\frac{1}{\overline{a_{1, i i}^{\varepsilon}}}(\omega, 0)
$$

which shows that $\tilde{T}_{i i}^{\varepsilon}(\omega,-L, 0)=T_{i i}^{\varepsilon}(\omega,-L, 0)$. Accordingly, the new incoming signal propagates into the medium $\left(\eta_{i i}, \nu_{i i}\right)$, and generates a new transmitted signal which we observe at the time $t_{2}+\varepsilon t$ that is around the time $t_{2}$ in the 
scale of the initial pulse. In terms of the transmission coefficients $T_{i i}^{\varepsilon}$ associated the process $\left(\eta_{i i}, \nu_{i i}\right)$, the observed transmitted signal is given by

$$
p_{t r(T R T)}\left(t_{2}+\varepsilon t, z=0\right)=\int \hat{p}_{i n c(T R T)}^{\varepsilon}(\omega) T_{i i}^{\varepsilon}(\omega,-L, 0) e^{\frac{i \omega t_{2}}{\varepsilon}+i \omega t-i \omega \frac{L}{\varepsilon}} d \omega .
$$

Substituting the expression of $\hat{p}_{\text {inc }(T R T)}^{\varepsilon}$ into this equation yields the following representation of the new transmitted signal

$$
\begin{aligned}
p_{t r(T R T)}\left(t_{2}+\varepsilon t, z=0\right) & =\iint e^{i \omega t} e^{\frac{i \omega\left(t_{2}-t_{1}-L\right)}{\varepsilon}} \bar{f}(\omega-\varepsilon h) \overline{\hat{G}}_{t_{0}, t_{1}}(h) \\
& \times T_{i i}^{\varepsilon}(\omega,-L, 0) \overline{T_{i}^{\varepsilon}}(\omega-\varepsilon h,-L, 0) d h d \omega .
\end{aligned}
$$

The precise asymptotics of the transmitted wave will be carried out in the next section. It is easily seen that the refocusing will only take place if $t_{2}=L+t_{1}$ due to the fast phase.

\section{The refocused pulse for Time Reversal in Reflection}

The refocused pulse is given by

$$
\begin{aligned}
p_{r e f(T R R)}\left(t_{1}+\varepsilon t,\right. & z=0)=\iint e^{i \omega t} e^{i \varepsilon h t / 2} \overline{\hat{f}}(\omega-\varepsilon h / 2) \overline{\hat{G}}_{t_{1}}(h) \\
& \times R_{i i}^{\varepsilon}(\omega+\varepsilon h / 2,-L, 0) \overline{R_{i}^{\varepsilon}}(\omega-\varepsilon h / 2,-L, 0) d h d \omega .
\end{aligned}
$$

This section is devoted to the proof of the convergence of the refocused pulse shape to an effective shape as $\varepsilon \rightarrow 0$.

\subsection{Tightness}

We first address the tightness of the process.

Lemma 7.1 The refocused pulse $\left(\left(p_{\text {ref(TRR) }}^{\varepsilon}\left(t_{1}+\varepsilon t\right)\right)_{-\infty<t<\infty}\right)_{\varepsilon>0}$ is a tight (i.e. weakly compact) family in the space of continuous trajectories equipped with the sup norm.

Proof. We must show that, for any $\delta>0$, there exists a compact subset $K$ of the space of continuous bounded functions such that:

$$
\sup _{\varepsilon>0} \mathbb{P}\left(p_{r e f(T R R)}^{\varepsilon}\left(t_{1}+\varepsilon \cdot\right) \in K\right) \geq 1-\delta
$$


On the one hand the energy conservation relation (16) yields that $p_{\text {ref(TRR) }}^{\varepsilon}\left(t_{1}+\right.$ $\varepsilon t$ ) is uniformly bounded by:

$$
\left|p_{\text {ref(TRR) }}^{\varepsilon}\left(t_{1}+\varepsilon t\right)\right| \leq \int|\hat{f}(\omega)| d \omega \times \int\left|\hat{G}_{t_{1}}(h)\right| d h
$$

On the other hand the modulus of continuity

$$
M^{\varepsilon}(\delta)=\sup _{\left|s_{1}-s_{2}\right| \leq \delta}\left|p_{r e f(T R R)}^{\varepsilon}\left(t_{1}+\varepsilon s_{1}\right)-p_{r e f(T R R)}^{\varepsilon}\left(t_{1}+\varepsilon s_{2}\right)\right|
$$

is bounded by

$$
M^{\varepsilon}(\delta) \leq \int \sup _{\left|s_{1}-s_{2}\right| \leq \delta}\left|1-\exp \left(i \omega\left(s_{1}-s_{2}\right)\right)\right||\hat{f}(\omega)| d \omega \times \int\left|\hat{G}_{t_{1}}(h)\right| d h
$$

which goes to zero as $\delta$ goes to zero uniformly with respect to $\varepsilon$.

\subsection{Convergence of the finite-dimensional distributions}

The uniform boundedness (25) implies that the finite-dimensional distributions of the process $p_{r e f(T R R)}^{\varepsilon}\left(t_{1}+.\right)$ will be characterized by the moments

$$
\mathbb{E}\left[p_{r e f(T R R)}^{\varepsilon}\left(t_{1}+\varepsilon s_{1}\right)^{p_{1}} \ldots p_{r e f(T R R)}^{\varepsilon}\left(t_{1}+\varepsilon s_{k}\right)^{p_{k}}\right]
$$

for every real numbers $s_{1}<\ldots<s_{k}$ and every integers $p_{1}, \ldots, p_{k}$.

First moment. Let us first address the first moment. Using the representation (24) the expectation of $p_{\text {ref(TRR) }}^{\varepsilon}\left(t_{1}+\varepsilon t\right)$ reads:

$$
\begin{aligned}
\mathbb{E}\left[p _ { \text { ref(TRR) } } \left(t_{1}\right.\right. & +\varepsilon t)]=\iint e^{i \omega t} e^{i \varepsilon h t / 2} \overline{\hat{f}}(\omega-\varepsilon h / 2) \overline{\hat{G}}_{t_{1}}(h) \\
& \times \mathbb{E}\left[R_{i i}^{\varepsilon}(\omega+\varepsilon h / 2,-L, 0) \overline{R_{i}^{\varepsilon}}(\omega-\varepsilon h / 2,-L, 0)\right] d h d \omega .
\end{aligned}
$$

As shown by (27), the statistical distribution of the refocused pulse will depend on the frequency autocorrelation function of the reflection coefficient. We shall extend the approach developed in [3,9]. It is necessary to consider a family of moments so as to get a closed system of equations. We introduce for $q, p \in \mathbb{N}$

$$
U_{q, p}^{\varepsilon}(\omega, h, z)=\left(R_{i i}^{\varepsilon}\left(\omega+\frac{\varepsilon h}{2},-L, z\right)\right)^{q}\left(\overline{R_{i}^{\varepsilon}}\left(\omega-\frac{\varepsilon h}{2},-L, z\right)\right)^{p} .
$$

Using the Riccati equation (14) satisfied by $R^{\varepsilon}$, we deduce 


$$
\begin{aligned}
\frac{\partial U_{q, p}^{\varepsilon}}{\partial z}= & i \omega\left(p m_{i}^{\varepsilon}-q m_{i i}^{\varepsilon}\right) U_{q, p}^{\varepsilon}+i e^{\frac{2 i \omega z}{\varepsilon}} \frac{\omega}{2}\left(-q n_{i i}^{\varepsilon} e^{i h z} U_{q-1, p}^{\varepsilon}+p n_{i}^{\varepsilon} e^{-i h z} U_{q, p+1}^{\varepsilon}\right) \\
& +i e^{-\frac{2 i \omega z}{\varepsilon}} \frac{\omega}{2}\left(p n_{i}^{\varepsilon} e^{i h z} U_{q, p-1}^{\varepsilon}-q n_{i i}^{\varepsilon} e^{-i h z} U_{q+1, p}^{\varepsilon}\right)
\end{aligned}
$$

starting from $U_{q, p}^{\varepsilon}(\omega, h, z=-L)=\mathbf{1}_{0}(q) \mathbf{1}_{0}(p)$, where $\mathbf{1}_{0}(q)=1$ if $q=0$ and 0 otherwise. Taking a shifted scaled Fourier transform with respect to $h$

$$
V_{q, p}^{\varepsilon}(\omega, \tau, z)=\frac{1}{2 \pi} \int e^{i h(\tau-(q+p) z)} U_{q, p}^{\varepsilon}(\omega, h, z) d h
$$

we get

$$
\begin{aligned}
& \frac{\partial V_{q, p}^{\varepsilon}}{\partial z}=-(q+p) \frac{\partial V_{q, p}^{\varepsilon}}{\partial \tau}+i \omega\left(p m_{i}^{\varepsilon}-q m_{i i}^{\varepsilon}\right) V_{q, p}^{\varepsilon} \\
& \quad+i \frac{\omega}{2} e^{\frac{2 i \omega z}{\varepsilon}}\left(-q n_{i i}^{\varepsilon} V_{q-1, p}^{\varepsilon}+p n_{i}^{\varepsilon} V_{q, p+1}^{\varepsilon}\right)+i \frac{\omega}{2} e^{-\frac{2 i \omega z}{\varepsilon}}\left(p n_{i}^{\varepsilon} V_{q, p-1}^{\varepsilon}-q n_{i i}^{\varepsilon} V_{q+1, p}^{\varepsilon}\right)
\end{aligned}
$$

starting from $V_{q, p}^{\varepsilon}(\omega, \tau, z=-L)=\delta_{0}(\tau) \mathbf{1}_{0}(q) \mathbf{1}_{0}(p)$. Applying a diffusionapproximation theorem [3, Section 3] establishes that the processes $V_{q, p}^{\varepsilon}$ converge to diffusion processes as $\varepsilon \rightarrow 0$. In particular the expectations $\mathbb{E}\left[V_{p, p}^{\varepsilon}(\omega, \tau, z)\right]$, $p \in \mathbb{N}$, converge to $v_{p}(\omega, \tau, z)$ which obey the closed system of transport equations

$$
\begin{aligned}
& \frac{\partial v_{p}}{\partial z}+2 p \frac{\partial v_{p}}{\partial \tau}=\left(\mathcal{L}_{\omega} v\right)_{p}-2 \alpha_{m}\left(1-\delta_{m}\right) \omega^{2} p^{2} v_{p} \\
& \left(\mathcal{L}_{\omega} \phi\right)_{p}=\frac{1}{2} \alpha_{n} \delta_{n} \omega^{2} p^{2}\left(\phi_{p+1}+\phi_{p-1}-2 \phi_{p}\right)-\alpha_{n}\left(1-\delta_{n}\right) \omega^{2} p^{2} \phi_{p}, \\
& v_{p}(\omega, \tau, z=-L)=\delta_{0}(\tau) \mathbf{1}_{0}(p),
\end{aligned}
$$

where

$$
\begin{aligned}
\alpha_{n} & =\int_{0}^{\infty} \mathbb{E}\left[n_{i}(0) n_{i}(z)\right] d z=\int_{0}^{\infty} \mathbb{E}\left[n_{i i}(0) n_{i i}(z)\right] d z \\
\alpha_{m} & =\int_{0}^{\infty} \mathbb{E}\left[m_{i}(0) m_{i}(z)\right] d z=\int_{0}^{\infty} \mathbb{E}\left[m_{i i}(0) m_{i i}(z)\right] d z \\
\delta_{n} & =\frac{1}{\alpha_{n}} \int_{0}^{\infty} \mathbb{E}\left[n_{i i}(0) n_{i}(z)\right] d z=\frac{1}{\alpha_{n}} \int_{0}^{\infty} \mathbb{E}\left[n_{i}(0) n_{i i}(z)\right] d z \\
\delta_{m} & =\frac{1}{\alpha_{m}} \int_{0}^{\infty} \mathbb{E}\left[m_{i i}(0) m_{i}(z)\right] d z=\frac{1}{\alpha_{m}} \int_{0}^{\infty} \mathbb{E}\left[m_{i}(0) m_{i i}(z)\right] d z .
\end{aligned}
$$

$\alpha_{m}$ and $\alpha_{n}$ are the standard integrated covariances of the processes $m$ and $n$ in absence of time perturbations. $\delta_{m}$, respectively $\delta_{n}$, characterizes the corre- 
lation degree between the processes $m_{i}$ and $m_{i i}$, respectively $n_{i}$ and $n_{i i}$. If the fluctuations of the medium are the same in the two steps of the experiment $\left(m_{i} \equiv m_{i i}, n_{i} \equiv n_{i i}\right)$, then $\delta_{m}=\delta_{n}=1$, and the limit transport equations (29) have the same form as the ones obtained in [3]. If the fluctuations of the medium are completely uncorrelated in the two steps of the experiment $\left(\left(\eta_{i}, \nu_{i}\right)\right.$ and $\left(\eta_{i i}, \nu_{i i}\right)$ independent), then $\delta_{m}=\delta_{n}=0$ so that $v_{p}(\omega, \tau, z)=\delta_{0}(\tau) \mathbf{1}_{0}(p)$.

We then get the limit of the autocorrelation function of the reflection coefficient:

$$
\mathbb{E}\left[R_{i i}^{\varepsilon}\left(\omega+\frac{\varepsilon h}{2},-L, 0\right) \overline{R_{i}^{\varepsilon}}\left(\omega-\frac{\varepsilon h}{2},-L, 0\right)\right] \stackrel{\varepsilon \rightarrow 0}{\longrightarrow} \int v_{1}(\omega, \tau, 0) e^{-i h \tau} d \tau
$$

The quantity $v_{1}(\omega, \tau, 0)$ is obtained through the system of transport equations (29). We can rewrite this system by introducing the family of processes

$$
\begin{aligned}
& d W_{p}+2 p \frac{\partial W_{p}}{\partial \tau} d z=2 i p \omega \sqrt{\alpha_{m}\left(1-\delta_{m}\right)} W_{p} \circ d B_{z}+\left(\mathcal{L}_{\omega} W\right)_{p} d z \\
& W_{p}(\omega, \tau, z=-L)=\delta_{0}(\tau) \mathbf{1}_{0}(p)
\end{aligned}
$$

driven by a standard Brownian motion $B_{z}$. Indeed it is straightforward to check that we have

$$
v_{p}(\omega, \tau, z)=\mathbf{E}\left[W_{p}(\omega, \tau, z)\right]
$$

where the expectation $\mathbf{E}$ is taken with respect to the distribution of the Brownian motion $B_{z}$. Using this expression of the asymptotic autocorrelation function yields

$$
\begin{aligned}
\mathbb{E}\left[p_{r e f(T R R)}\left(t_{1}+\varepsilon t\right)\right] & \stackrel{\varepsilon \rightarrow 0}{\longrightarrow} \iiint e^{i \omega t} e^{-i h \tau} \overline{\hat{f}}(\omega) \overline{\hat{G}_{t_{1}}}(h) \mathbf{E}\left[W_{1}(\omega, \tau, 0)\right] d h d \tau d \omega \\
& =\iint e^{i \omega t} \overline{\hat{f}}(\omega) G_{t_{1}}(\tau) \mathbf{E}\left[W_{1}(\omega, \tau, 0)\right] d \tau d \omega .
\end{aligned}
$$

Higher order moments. Let us now consider the general moment (26). Using the representation (24) for each factor $p_{\text {ref(TRR) }}^{\varepsilon}\left(t_{1}+\varepsilon s_{j}\right)$, these moments can be written as multiple integrals over $p=\sum_{j=1}^{k} p_{j}$ frequencies:

$$
\begin{gathered}
\mathbb{E}\left[p_{\text {ref(TRR) }}^{\varepsilon}\left(t_{1}+\varepsilon s_{1}\right)^{p_{1}} \ldots p_{\text {ref(TRR) }}^{\varepsilon}\left(t_{1}+\varepsilon s_{k}\right)^{p_{k}}\right]= \\
\int \ldots \int \mathbb{E}\left[\prod_{\substack{1 \leq j \leq k \\
1 \leq l \leq p_{j}}} U_{1,1}^{\varepsilon}\left(\omega_{j, l}, h_{j, l}, 0\right)\right] \prod_{\substack{1 \leq j \leq k \\
1 \leq l \leq p_{j}}} \overline{\hat{f}}\left(\omega_{j, l}\right) e^{i \omega_{j, l} s_{j}} e^{i \varepsilon h_{j, l} s_{j} / 2} \overline{\hat{G}_{t_{1}}}\left(h_{j, l}\right) d \omega_{j, l} d h_{j, l}
\end{gathered}
$$


The important quantity is $\mathbb{E}\left[\prod_{j, l} U_{1,1}^{\varepsilon}\left(\omega_{j, l}, h_{j, l}, 0\right)\right]$. Our problem is now to find the limit, as $\varepsilon$ goes to 0 , of these moments for $k$ distinct frequencies. This limit will be deduced from the study of the convergence of the distribution of $\left(U_{q_{1}, p_{1}}^{\varepsilon}\left(\omega_{1}, h_{1}, z\right), \ldots, U_{q_{k}, p_{k}}^{\varepsilon}\left(\omega_{k}, h_{k}, z\right)\right)$ which results once again from the application of a diffusion-approximation theorem. Introducing $V^{\varepsilon}$ as in Eq. (28) it is found that $\left(V_{q_{1}, p_{1}}^{\varepsilon}\left(\omega_{1}, \tau_{1}, z\right), \ldots, V_{q_{k}, p_{k}}^{\varepsilon}\left(\omega_{k}, \tau_{k}, z\right)\right)$ converges as $\varepsilon \rightarrow 0$ to a diffusion process. In particular,

$$
v_{p_{1}, \ldots, p_{k}}\left(\omega_{1}, \ldots, \omega_{k}, \tau_{1}, \ldots, \tau_{k}, z\right):=\lim _{\varepsilon \rightarrow 0} \mathbb{E}\left[\prod_{j} V_{p_{j}, p_{j}}^{\varepsilon}\left(\omega_{j}, \tau_{j}, z\right)\right]
$$

is solution of

$$
\begin{aligned}
& \frac{\partial v_{p_{1}, \ldots, p_{k}}}{\partial z}+2 \sum_{j} p_{j} \frac{\partial v_{p_{1}, \ldots, p_{k}}}{\partial \tau_{j}}=\sum_{j} \mathcal{L}_{\omega_{j}} v_{p_{1}, \ldots, p_{k}}-2 \alpha_{m}\left(1-\delta_{m}\right)\left(\sum_{j} \omega_{j} p_{j}\right)^{2} v_{p_{1}, \ldots, p_{k}} \\
& v_{p_{1}, \ldots, p_{k}}\left(\omega_{1}, \ldots, \omega_{k}, \tau_{1}, \ldots, \tau_{k}, z=-L\right)=\prod_{j} \delta_{0}\left(\tau_{j}\right) \mathbf{1}_{0}\left(p_{j}\right)
\end{aligned}
$$

Using the families of processes $W$ introduced in Eq. (36) defined for every frequency $\omega$ with the same Brownian motion $B_{z}$, a direct calculation shows that $\mathbf{E}\left[\prod_{j} W_{p_{j}}\left(\omega_{j}, \tau_{j}\right)\right]$ satisfies the above system, so that we have

$$
v_{p_{1}, \ldots, p_{k}}\left(\omega_{1}, \ldots, \omega_{k}, \tau_{1}, \ldots, \tau_{k}, z\right)=\mathbf{E}\left[\prod_{j} W_{p_{j}}\left(\omega_{j}, \tau_{j}, z\right)\right]
$$

and consequently,

$$
\begin{aligned}
& \mathbb{E}\left[p_{r e f(T R R)}^{\varepsilon}\left(t_{1}+\varepsilon s_{1}\right)^{p_{1}} \ldots p_{r e f(T R R)}^{\varepsilon}\left(t_{1}+\varepsilon s_{k}\right)^{p_{k}}\right] \stackrel{\varepsilon \rightarrow 0}{\longrightarrow} \\
& \int \ldots \int \mathbf{E}\left[\prod_{\substack{1 \leq j \leq k \\
1 \leq l \leq p_{j}}} W_{1}\left(\omega_{j, l}, \tau_{j, l}, 0\right)\right] \prod_{\substack{1 \leq j \leq k \\
1 \leq l \leq p_{j}}} \bar{f}\left(\omega_{j, l}\right) e^{i \omega_{j, l} s_{j}} G_{t_{1}}\left(\tau_{j, l}\right) d \omega_{j, l} d \tau_{j, l} \\
& =\mathbf{E}\left[\prod_{1 \leq j \leq k}\left(\int W_{1}(\omega, \tau, 0) \overline{\hat{f}}(\omega) e^{i \omega s_{j}} G_{t_{1}}(\tau) d \omega d \tau\right)^{p_{j}}\right] .
\end{aligned}
$$

This shows the convergence of the finite-dimensional distributions of $\left(p_{\text {ref(TRR) }}^{\varepsilon}\left(t_{1}+\right.\right.$ $\varepsilon s))_{s \in(-\infty, \infty)}$ to the ones of

$$
\int W_{1}(\omega, \tau, 0) \overline{\hat{f}}(\omega) e^{i \omega s} G_{t_{1}}(\tau) d \omega d \tau
$$




\subsection{Convergence of the refocused pulse}

We have just proved the tightness of the process $\left(p_{\text {ref }(T R R)}\left(t_{1}+\varepsilon t, z=0\right)\right)_{t \in(-\infty, \infty)}$ as well as the convergence of its finite-dimensional distributions. Accordingly this demonstrates the following theorem.

Theorem 7.1 The refocused signal $\left(p_{\text {ref }(T R R)}\left(t_{1}+\varepsilon t, z=0\right)\right)_{t \in(-\infty, \infty)}$ converges in distribution as $\varepsilon \rightarrow 0$ to

$$
P_{r e f(T R R)}(t)=\int \Lambda_{r e f}^{L}(\omega, \tau) \overline{\hat{f}}(\omega) e^{i \omega t} G_{t_{1}}(\tau) d \omega d \tau
$$

where $\Lambda_{\text {ref }}^{L}(\omega, \tau)=W_{1}(\omega, \tau, 0)$ is the random density given by the system (36). We can also write that

$$
P_{r e f(T R R)}(t)=\left(f(-\cdot) * K_{T R R}(\cdot)\right)(t) .
$$

The Fourier transform of the kernel $K_{T R R}$ is the convolution of the timeinverted cut-off function $G_{t_{1}}$ with the density $\tau \mapsto \Lambda_{\text {ref }}^{L}(\omega, \tau)$ evaluated at 0 :

$$
\hat{K}_{T R R}(\omega)=\left(G_{t_{1}}(-\cdot) * \Lambda_{r e f}^{L}(\omega, \cdot)\right)(0)=\int G_{t_{1}}(\tau) \Lambda_{r e f}^{L}(\omega, \tau) d \tau
$$

We can give a probabilistic representation of the density $\Lambda_{\text {ref }}^{L}$ in terms of a jump Markov process. Let us introduce the process $\left(X_{t}\right)_{t \geq 0}$ with state space $\mathbb{N} \cup\{\partial\}$ (where $\partial$ is the cemetery state) and infinitesimal generator $\mathcal{L}_{\omega}$ given by (30). Note that, as soon as $\delta_{n}<1$, the jump process can be killed. When the jump process reaches the state $x \in \mathbb{N}^{*}$, a random clock with exponential distribution and mean $\tau(\omega, x)=\left(x^{2} \alpha_{n} \omega^{2}\right)^{-1}$ starts running. When the clock strikes, the process is killed and goes to $\partial$ with probability $p_{\partial}=1-\delta_{n}$, it jumps to $x+1$ with probability $p_{+}=\delta_{n} / 2$, and to $x-1$ with probability $p_{-}=p_{+}$. Finally, 0 is an absorbing state. This representation of a system of transport equations in terms of a canonical Markov process was first introduced in [3], and it turns out that we can extend this idea to the system (36) by means of a Feynman-Kac formula. After some algebra we get

$$
\begin{aligned}
\int_{\tau_{0}}^{\tau_{1}} W_{1}(\omega, \tau, 0) d \tau=\mathbb{E}_{1} & {\left[\mathbf{1}_{\int_{0}^{L} 2 X_{s} d s \in\left[\tau_{0}, \tau_{1}\right]} \mathbf{1}_{X_{L}=0}\right.} \\
& \left.\times \exp \left(2 i \sqrt{\alpha_{m}\left(1-\delta_{m}\right)} \omega \int_{0}^{L} X_{s} d B_{L-s}\right)\right]
\end{aligned}
$$

where $\mathbb{E}_{x_{0}}$ stands for the expectation over the distribution of the jump process starting from $X_{0}=x_{0}$ (the expectation is not taken with respect to the 
distribution of the Brownian motion $B$ ). The refocused pulse shape has thus a random shape in presence of time perturbations of the medium fluctuations. There is no more statistical stability as there used to be in the standard configurations where the medium is time-independent [11].

\subsection{Pulse stabilization for a particular class of perturbations}

The main result of this section is the refocusing of the pulse with a deterministic shape in the particular case $\delta_{m}=0$. The process $W_{1}$ is then deterministic, hence the density $\Lambda_{r e f}^{L}$ is also deterministic. The configuration $\delta_{m}=1$ occurs when the time-perturbation affects both the density $\rho$ (through the process $\nu$ ) and the bulk modulus $K$ (through the process $\eta$ ), but the process $m=\eta+\nu$ is not perturbed so that $m_{i}=m_{i i}$. This means roughly speaking that the local velocity is not affected by the time-perturbation. For instance this occurs if $\eta=-\nu$, since this implies $m_{i}=m_{i i} \equiv 0$.

Theorem 7.2 If $\delta_{m}=1$, then the signal $\left(p_{\text {ref }(T R R)}\left(t_{1}+\varepsilon t, z=0\right)\right)_{t \in(-\infty, \infty)}$ converges in probability to $P_{\text {ref(TRR) }}(t)$ as $\varepsilon \rightarrow 0$ where $P_{\text {ref(TRR) }}$ is the deterministic pulse shape:

$$
P_{r e f(T R R)}(t)=\left(f(-\cdot) * K_{T R R}(\cdot)\right)(t) .
$$

The Fourier transform of the kernel $K_{T R R}$ is (37).

\subsection{Analysis of the refocused pulse shape}

The explicit description of the refocused pulse shape allows us to perform explicit calculations. For instance, we can compute the mean pulse shape:

$$
\begin{gathered}
\mathbb{E}\left[P_{r e f(T R R)}(t)\right]=\left(f(-\cdot) * \mathbb{E}\left[K_{T R R}\right](\cdot)\right)(t) \\
\mathbb{E}\left[\hat{K}_{T R R}\right](\omega)=\int G_{t_{1}}(\tau) \mathbb{E}\left[\Lambda_{r e f}^{L}\right](\omega, \tau) d \tau
\end{gathered}
$$

We shall now address the case of a semi-finite slab $L \rightarrow \infty$. This case leads to some simple analytical results. In particular, long but straightforward calculations establish that

$$
\mathbb{E}\left[W_{n}\right](\omega, \tau, 0)=\frac{\partial}{\partial \tau}\left[\left(\frac{\delta_{0} \tanh \left(\frac{\sqrt{1-\delta_{0}^{2}} \alpha_{0} \omega^{2} \tau}{4}\right)}{\sqrt{1-\delta_{0}^{2}}+\tanh \left(\frac{\sqrt{1-\delta_{0}^{2}} \alpha_{0} \omega^{2} \tau}{4}\right)}\right)^{n} \mathbf{1}_{[0, \infty)}(\tau)\right]
$$


where

$$
\alpha_{0}=\alpha_{n}+2\left(1-\delta_{m}\right) \alpha_{m}, \quad \delta_{0}=\frac{\delta_{n} \alpha_{n}}{\alpha_{n}+2\left(1-\delta_{m}\right) \alpha_{m}}
$$

We thus get a closed form expression of the expectation of the density

$$
\mathbb{E}\left[\Lambda_{r e f}^{L \rightarrow \infty}\right](\omega, \tau)=\frac{\alpha_{0} \omega^{2} \delta_{0}}{4} \frac{1-\tanh ^{2}\left(\frac{\sqrt{1-\delta_{0}^{2}} \alpha_{0} \omega^{2} \tau}{4}\right)}{\left[1+\frac{1}{\sqrt{1-\delta_{0}^{2}}} \tanh \left(\frac{\sqrt{1-\delta_{0}^{2}} \alpha_{0} \omega^{2} \tau}{4}\right)\right]^{2}} \mathbf{1}_{[0, \infty)}(\tau)
$$

Note that in the particular case $\delta_{m}=1$, we are actually discussing the deterministic convolution kernel that gives the refocused pulse shape. In that case $\delta_{0}=\delta_{n}$ and $\alpha_{0}=\alpha_{n}$ and all the expectations can be removed from the forthcoming expressions.

Total record. Assume that we record everything at the mirror, so that $G_{t_{1}} \equiv$ 1. In these conditions $\mathbb{E}\left[\hat{K}_{T R R}\right](\omega)=G_{T R R}$ where

$$
G_{T R R}=\frac{1-\sqrt{1-\delta_{0}^{2}}}{\delta_{0}}
$$

If the medium is constant during the time reversal experiment $\left(\eta_{i}, \nu_{i}\right)=$ $\left(\eta_{i i}, \nu_{i i}\right)$, then $\delta_{n}=\delta_{m}=1$ and $\hat{K}_{T R R}(\omega) \equiv 1$. This is of course expected: The pulse has been completely scattered back by the random half-space due to Anderson localization [19], and we have sent back everything that has been recorded, so we get a perfect refocusing as a result of the time-reversibility of the wave equation.

If the medium has changed between the two parts of the experiment, then $\mathbb{E}\left[\hat{K}_{T R R}\right](\omega)$ is equal to the constant $G_{T R R}$ that is strictly less than 1 . This means that the mean refocused pulse has exactly the original pulse shape, up to the attenuation factor $G_{T R R}$ that depends only on the correlation degree $\delta_{0}$. Note that in this configuration all frequency modes are reflected back whatever the realization of the medium due to the localization effect. The attenuation factor $G_{T R R}$ means that, in the case of a time-dependent random medium, only a part of the reflected wave contributes to the refocused pulse shape. The attenuation factor $G_{T R R}$ is very sensitive to a change of the medium: a mismatch of $1 \%$ (resp. 5\%) leads to a reduction of $13 \%$ (resp. 28\%) of the amplitude of the refocused pulse (see figure 4a).

Partial record. Assume now that we record only a piece of the reflected signal at the mirror, with a square cut-off function $G_{t_{1}}(t)=\mathbf{1}_{\left[0, t_{1}\right]}(t)$. If the 
a)

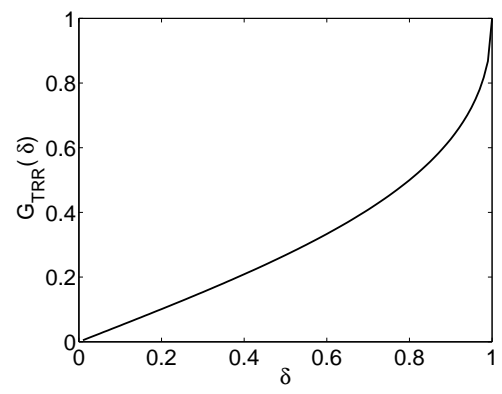

b)

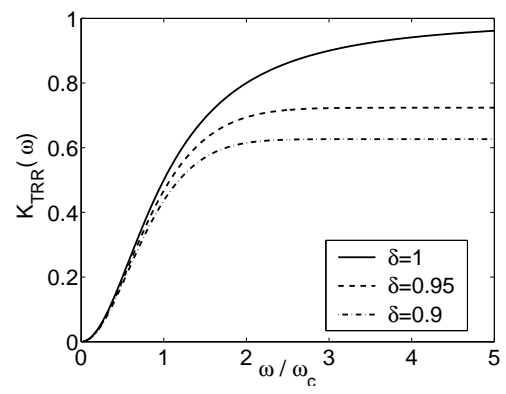

Fig. 4. Picture a: Attenuation factor $G_{T R R}$ as a function of the correlation degree $\delta$ (total record). Picture b: Mean filter $\mathbb{E}\left[\hat{K}_{T R R}\right]$ as a function of the frequency $\omega$ for different correlation degrees (partial record, square cut-off function $\left.G_{t_{1}}(t)=\mathbf{1}_{\left[0, t_{1}\right]}(t)\right)$. Here $\omega_{c}=2 / \sqrt{\alpha_{0} t_{1}}$.

medium is constant during the time reversal experiment $\left(\eta_{i}, \nu_{i}\right)=\left(\eta_{i i}, \nu_{i i}\right)$, then $\delta_{m}=\delta_{n}=1$ and

$$
\hat{K}_{T R R}(\omega)=\frac{\alpha_{n} \omega^{2} t_{1}}{4+\alpha_{n} \omega^{2} t_{1}}
$$

The refocused pulse is a filtered version of the original pulse shape with a highband filter. The cut-off frequency of the filter is $\omega_{c}=2 / \sqrt{\alpha_{n} t_{1}}$. If the medium has changed between the two parts of the experiment, then $\mathbb{E}\left[\hat{K}_{T R R}\right](\omega)$ is a function of $\alpha_{0} \omega^{2} t_{1}$ and $\delta_{0}$ :

$$
\mathbb{E}\left[\hat{K}_{T R R}\right](\omega)=\delta_{0} \frac{\tanh \left(\frac{\sqrt{1-\delta_{0}^{2}} \alpha_{0} \omega^{2} t_{1}}{4}\right)}{\sqrt{1-\delta_{0}^{2}}+\tanh \left(\frac{\sqrt{1-\delta_{0}^{2}} \alpha_{0} \omega^{2} t_{1}}{4}\right)}
$$

For low frequencies $\omega \ll \omega_{c}$, we have $\mathbb{E}\left[\hat{K}_{T R R}\right](\omega) \simeq \delta_{0} \alpha_{0} \omega^{2} t_{1} / 4$ which means that we have a reduction by a factor $\delta_{0}$ w.r.t. the constant configuration. Accordingly, if the frequency content of the initial pulse is in the low-frequency band (below $\omega_{c}$ ), then the refocused pulse has the original pulse shape, up to the attenuation factor $\delta_{0}$.

For high frequencies $\omega \gg \omega_{c}$, we have $\mathbb{E}\left[\hat{K}_{T R R}\right](\omega) \simeq G_{T R R}$ that is to say we recover the case of the semi-infinite slab. This is not surprising: the highfrequencies are those which are scattered back very early, at the beginning of the slab, so they probe the slab as if it were semi-infinite. Note that the attenuation factor for high-frequencies (plotted in Fig. 4a) is much stronger than the attenuation factor for low frequencies (which is simply $\delta_{n}$ ). This can be seen in Fig. 4b where the low frequencies are almost recovered as in the time-independent case, while the high-frequencies are dramatically attenuated. Once again, the high-frequencies are reflected back, but part of this reflected wave does not contribute coherently to the refocused pulse shape. 


\section{The refocused pulse for Time Reversal in Transmission}

We first study the autocorrelation function of the transmission coefficient at two nearby frequencies. We define a family of processes indexed by $q, p \in \mathbb{N}$

$$
\tilde{U}_{q, p}^{\varepsilon}(\omega, h, z)=U_{q, p}^{\varepsilon}(\omega, h, z) T_{i i}^{\varepsilon}\left(\omega+\frac{\varepsilon h}{2},-L, z\right) \overline{T_{i}^{\varepsilon}}\left(\omega-\frac{\varepsilon h}{2},-L, z\right)
$$

Taking a shifted scaled Fourier transform with respect to $h$

$$
\tilde{V}_{q, p}^{\varepsilon}(\omega, \tau, z)=\frac{1}{2 \pi} \int e^{i h(\tau-(q+p) z)} \tilde{U}_{q, p}^{\varepsilon}(\omega, h, z) d h
$$

we get that $\left(\tilde{V}_{q, p}^{\varepsilon}\right)_{q, p \in \mathbb{N}}$ satisfy the system of ordinary differential equations

$$
\begin{aligned}
\frac{\partial \tilde{V}_{q, p}^{\varepsilon}}{\partial z}= & -(q+p) \frac{\partial \tilde{V}_{q, p}^{\varepsilon}}{\partial \tau}+i \omega\left(\left(p+\frac{1}{2}\right) m_{i}^{\varepsilon}-\left(q+\frac{1}{2}\right) m_{i i}^{\varepsilon}\right) \tilde{V}_{q, p}^{\varepsilon} \\
& +i e^{\frac{2 i \omega z}{\varepsilon}} \frac{\omega}{2}\left(-q n_{i i}^{\varepsilon} \tilde{V}_{q-1, p}^{\varepsilon}+(p+1) n_{i}^{\varepsilon} \tilde{V}_{q, p+1}^{\varepsilon}\right) \\
& +i e^{-\frac{2 i \omega z}{\varepsilon}} \frac{\omega}{2}\left(p n_{i}^{\varepsilon} \tilde{V}_{q, p-1}^{\varepsilon}-(q+1) n_{i i}^{\varepsilon} \tilde{V}_{q+1, p}^{\varepsilon}\right)
\end{aligned}
$$

starting from $\tilde{V}_{q, p}^{\varepsilon}(\omega, \tau, z=-L)=\delta_{0}(\tau) \mathbf{1}_{0}(q) \mathbf{1}_{0}(p)$. Applying a diffusionapproximation theorem [3, Section 3.14] establishes that the processes $\tilde{V}_{q, p}^{\varepsilon}$ converge to diffusion processes as $\varepsilon \rightarrow 0$. In particular the expectations $\mathbb{E}\left[\tilde{V}_{p, p}^{\varepsilon}(\omega, \tau, z)\right]$ converge to $\tilde{v}_{p}(\omega, \tau, z)$ which obey the closed system of transport equations

$$
\frac{\partial \tilde{v}_{p}}{\partial z}+2 p \frac{\partial \tilde{v}_{p}}{\partial \tau}=\left(\tilde{\mathcal{L}}_{\omega} \tilde{v}\right)_{p}-2 \alpha_{m}\left(1-\delta_{m}\right) \omega^{2}\left(p+\frac{1}{2}\right)^{2} \tilde{v}_{p}
$$

starting from $\tilde{v}_{p}(\omega, \tau, z=-L)=\delta_{0}(\tau) \mathbf{1}_{0}(p)$, where

$$
\begin{aligned}
\left(\tilde{\mathcal{L}}_{\omega} \phi\right)_{p}= & \frac{1}{2} \alpha_{n} \delta_{n} \omega^{2}\left((p+1)^{2}\left(\phi_{p+1}-\phi_{p}\right)+p^{2}\left(\phi_{p-1}-\phi_{p}\right)\right) \\
& -\frac{1}{2} \alpha_{n}\left(1-\delta_{n}\right) \omega^{2}\left((p+1)^{2}+p^{2}\right) \phi_{p}
\end{aligned}
$$

We then get the limit of the autocorrelation function of the transmission coefficient:

$$
\mathbb{E}\left[T_{i i}^{\varepsilon}\left(\omega+\frac{\varepsilon h}{2},-L, 0\right) \overline{T_{i}^{\varepsilon}}\left(\omega-\frac{\varepsilon h}{2},-L, 0\right)\right] \stackrel{\varepsilon \rightarrow 0}{\longrightarrow} \int \tilde{v}_{0}(\omega, \tau, 0) e^{-i h \tau} d \tau
$$

Applying the same strategy as in Section 7, we introduce the family of pro- 
cesses $\tilde{W}_{p}$ that satisfy the system driven by a single Brownian motion $B$,

$$
d \tilde{W}_{p}+2 p \frac{\partial \tilde{W}_{p}}{\partial \tau} d z=2 i\left(\frac{1}{2}+p\right) \omega \sqrt{\alpha_{m}\left(1-\delta_{m}\right)} \tilde{W}_{p} \circ d B_{z}+\tilde{\mathcal{L}}_{\omega} \tilde{W}_{p} d z
$$

starting from $\tilde{W}_{p}(\omega, \tau, z=-L)=\delta_{0}(\tau) \mathbf{1}_{0}(p)$. We finally get the following result.

Theorem 8.1 The refocused signal $\left(p_{\text {ref(TRT) }}\left(t_{1}+L+\varepsilon t, z=0\right)\right)_{t \in(-\infty, \infty)}$ converges in distribution as $\varepsilon \rightarrow 0$ to

$$
P_{r e f(T R T)}(t)=\left(f(-\cdot) * K_{T R T}(\cdot)\right)(t)
$$

where the Fourier transform of the kernel $K_{T R T}$ is

$$
\hat{K}_{T R T}(\omega)=\int G_{t_{0}, t_{1}}(\tau) \Lambda_{t r}^{L}(\omega, d \tau)
$$

$\Lambda_{t r}^{L}(\omega, d \tau)=\tilde{W}_{0}(\omega, d \tau, 0)$ is the random measure given by the system (45).

We can interpret the transport equation in terms of a jump Markov process. Let us introduce the process $\left(\tilde{X}_{t}\right)_{t \geq 0}$ with state space $\mathbb{N} \cup \partial$ and infinitesimal generator $\tilde{\mathcal{L}}$ given by $(44)$. When the jump process reaches the state $x \in \mathbb{N}^{*}$, a random clock with exponential distribution and mean $\tau(\omega, x)=$ $2\left[\alpha_{n} \omega^{2}\left((x+1)^{2}+x^{2}\right)\right]^{-1}$ starts running. When the clock strikes, the process is killed and goes to $\partial$ with probability $p_{\partial}=1-\delta_{n}$, it jumps to $x+1$ with probability $p_{+, x}=\delta_{n} \frac{(x+1)^{2}}{(x+1)^{2}+x^{2}}$, finally it jumps to $n-1$ with probability $p_{-, x}=\delta_{n} \frac{x^{2}}{(x+1)^{2}+x^{2}}$. We have

$$
\begin{aligned}
\int_{\tau_{0}}^{\tau_{1}} \tilde{W}_{0}(\omega, d \tau, 0)=\tilde{\mathbb{E}}_{0} & {\left[\mathbf{1}_{\int_{0}^{L} 2 \tilde{X}_{s} d s \in\left[\tau_{0}, \tau_{1}\right]} \mathbf{1}_{\tilde{X}_{L}=0}\right.} \\
& \left.\times \exp \left(2 i \omega \sqrt{\alpha_{m}\left(1-\delta_{m}\right)} \int_{0}^{L}\left(\frac{1}{2}+\tilde{X}_{s}\right) d B_{L-s}\right)\right]
\end{aligned}
$$

where $\tilde{\mathbb{E}}_{x_{0}}$ stands for the expectation over the distribution of the jump process starting from $\tilde{X}_{0}=x_{0}$. It should be noted that $\tilde{W}_{0}$ is not a density with respect the Lebesgue measure over $\mathbb{R}^{+}$(while $W_{1}$ is a density as seen in Section 7 ). It consists actually of the sum of a Dirac mass at 0 and a density:

$$
\tilde{W}_{0}(\omega, d \tau, 0)=p_{\omega, d} \delta_{0}(d \tau)+\tilde{W}_{0, c}(\omega, \tau, 0) d \tau
$$


This expression is obtained by disintegrating the right-hand side of Eq. (48) over the first jump time of the process $(\tilde{X})_{t \geq 0}$. The weight of the Dirac mass is

$$
p_{\omega, d}=\exp \left(-\frac{\alpha_{n} \omega^{2} L}{2}+i \omega \sqrt{\alpha_{m}\left(1-\delta_{m}\right)} B_{L}\right)
$$

This part of the kernel results from the double action of the ODA theory on the front pulse. The pulse spreading is deterministic, but the fluctuations of the medium have changed so that we observe a random time delay equal to

$$
T_{d}=\sqrt{\alpha_{m}\left(1-\delta_{m}\right)} B_{L}
$$

The absolutely continuous part is given by

$$
\begin{gathered}
\int_{0}^{\tau_{1}} \tilde{W}_{0, c}(\omega, \tau, 0) d \tau=\int_{0}^{L} d t \exp \left(-\frac{\alpha_{n} \omega^{2}(L-t)}{2}+i \omega \sqrt{\alpha_{m}\left(1-\delta_{m}\right)}\left(B_{L}-B_{t}\right)\right) \\
\times \frac{\alpha_{n} \delta_{n} \omega^{2}}{2} \tilde{\mathbb{E}}_{1}\left[\mathbf{1}_{\int_{0}^{t} 2 \tilde{X}_{s} d s \in\left[0, \tau_{1}\right]} \mathbf{1}_{\tilde{X}_{t}=0} \exp \left(2 i \omega \sqrt{\alpha_{m}\left(1-\delta_{m}\right)} \int_{0}^{t}\left(\frac{1}{2}+\tilde{X}_{s}\right) d B_{t-s}\right)\right] .
\end{gathered}
$$

It does not seem possible to derive a closed form expression for the continuous part. We can either derive expansions, or perform numerical simulations based on Monte-Carlo simulations of the random jump process $\left(\tilde{X}_{t}\right)_{t \geq 0}$.

Finally, we state that pulse stabilization can be obtained for a particular class of perturbations. The following theorem expresses the self-averaging property of the refocused pulse in the case $\delta_{m}=1$.

Theorem 8.2 If $\delta_{m}=1$, then the signal $\left(p_{\operatorname{tr}(T R T)}\left(t_{1}+L+\varepsilon t, z=0\right)\right)_{t \in(-\infty, \infty)}$ converges in probability to $P_{\operatorname{tr}(T R T)}(t)$ as $\varepsilon \rightarrow 0$ where $P_{\operatorname{tr}(T R T)}$ is the deterministic refocused pulse shape (46).

Let us analyze the refocused pulse in the relevant configuration where we record the complete transmitted pulse so that we may assume that $G_{t_{0}, t_{1}} \equiv 1$. The kernel $K_{T R T}$ can then be split into two different parts

$$
\hat{K}_{T R T}(\omega)=\hat{K}_{T R T, d}(\omega)+\hat{K}_{T R T, c}(\omega)
$$

where $\hat{K}_{T R T, d}(\omega)=p_{\omega, d}$ is the contribution of the recorded front pulse (see Eq. (49) and $\hat{K}_{T R T, c}$ is the contribution of the recorded coda

$$
\hat{K}_{T R T, c}(\omega)=\int_{0}^{\infty} \tilde{W}_{0, c}(\omega, \tau, 0) d \tau
$$


The recorded front pulse and its contribution to the refocused pulse do not depend on the correlation degree between the media $\left(\eta_{i}, \nu_{i}\right)$ and $\left(\eta_{i i}, \nu_{i i}\right)$, up to a random time delay. This is expected: The pulse spreading described by the ODA theory is deterministic, it depends only on the statistical distribution of the random fluctuations of the medium, and not on the particular realization. The contribution of the coda is very sensitive to the correlation degree.

We can compute more precisely the expectation of the convolution kernel $K_{T R T}$. This kernel imposes the mean refocused pulse shape (which is deterministic in the particular framework $\left.\delta_{m}=1\right)$. We find that the expectation of the kernel $\mathbb{E}\left[\hat{K}_{T R T}\right](\omega)=\mathbb{E}\left[\hat{K}_{T R T, d}\right](\omega)+\mathbb{E}\left[\hat{K}_{T R T, c}\right](\omega)$ is a function of $\delta_{0}$ and $\alpha_{0} \omega^{2} L$. The contribution of the recorded front pulse is a Gaussian kernel

$$
\mathbb{E}\left[\hat{K}_{T R T, d}\right](\omega)=\exp \left(-\frac{\tilde{\alpha}_{0} \omega^{2} L}{2}\right)
$$

where $\tilde{\alpha}_{0}=\alpha_{n}+\left(1-\delta_{m}\right) \alpha_{m}$. The contribution of the recorded coda $\mathbb{E}\left[K_{T R T, c}\right]$ is a medium-band filter. The optimal frequency of the filter is $2 / \sqrt{\alpha_{n} L}$ in the constant medium configuration $\delta_{m}=\delta_{n}=1$ and the expectation of the kernel $\hat{K}_{T R T, c}$ reads as:

$$
\left.\mathbb{E}\left[\hat{K}_{T R T, c}\right](\omega)\right|_{\delta_{m}=\delta_{n}=1}=\frac{4}{\sqrt{\pi}} e^{-\frac{\alpha_{n} \omega^{2} L}{8}} \int_{0}^{\infty} \frac{x^{2} e^{-x^{2}}}{\cosh \left(\sqrt{\alpha_{n} \omega^{2} L / 2} x\right)} d x-e^{-\frac{\alpha_{n} \omega^{2} L}{2}} .
$$

As the correlation degree decreases, both the optimal frequency and the gain of the filter decay (see Fig. 5). $\mathbb{E}\left[\hat{K}_{T R T}\right](\omega)$ possesses a complicated expression. Introducing the tridiagonal matrix $\tilde{M}$ with entries $\tilde{M}_{j, j+1}=\tilde{M}_{j+1, j}=j^{2} \delta_{n} \alpha_{n}$, $\tilde{M}_{j, j}=(2 j-1)^{2} \tilde{\alpha}_{0}-2 j(j-1) \alpha_{n}$, and $\tilde{M}_{i, j}=0$ if $|i-j| \geq 2$, the expectation of the kernel $\hat{K}_{T R T}$ is given by

$$
\mathbb{E}\left[\hat{K}_{T R T}\right](\omega)=\left(\exp \left(\tilde{M} \frac{\omega^{2} L}{2}\right)\right)_{11} .
$$

Figure 5 has been obtained by a numerical evaluation of this exponential matrix. It appears that low frequencies are much less attenuated by time perturbations of the medium than high frequencies.

\section{$9 \quad$ Numerical experiments}

The acoustic equations are solved by a Lagrangian method along characteristics. The method is very efficient and gives place to exact radiation conditions at the ends of the computational domain. We briefly describe this method, but details will be presented elsewhere [2]. First the acoustic system (1) is written 


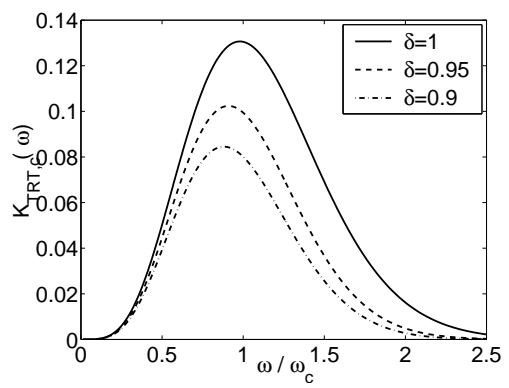

Fig. 5. Mean filter $\mathbb{E}\left[\hat{K}_{T R T, c}\right]$ as a function of the frequency $\omega$ for different correlation degrees (total record). Here $\delta_{m}=1, \delta_{n}=\delta, \omega_{c}=2 / \sqrt{\alpha_{n} L}$.

in the form $\left(\partial_{t}+\partial_{x}\right) A=-r B, \quad\left(\partial_{t}-\partial_{x}\right) B=r A$. Note that the characteristics for this system are straight lines. The right and left going modes are $A=\zeta^{1 / 2} u+\zeta^{1 / 2} p$ and $B=-\zeta^{1 / 2} u+\zeta^{1 / 2} p$. The impedance $\zeta=(\rho(x) K(x))^{1 / 2}$ is written as a function of the travel time $x=\int_{0}^{z} d s / c(s)$, where the acoustic sound speed is $c(x)=(K(x) / \rho(x))^{1 / 2}$. The right and left modes are coupled through the reflectivity $r(x)=0.5 \partial_{x} \zeta(x) / \zeta(x)$. The advection operators $\left(\partial_{t} \pm \partial_{x}\right)$ are discretized along characteristics using central differences. The evolution scheme takes the form $\vec{v}^{(n+1)}=M \vec{v}^{(n)}$ where $\vec{v}=(A, B)^{T}$. In [2] it is shown that $M$ is orthogonal and therefore the scheme is conservative. The mesh is defined in travel time and time. The computational domain is composed by a long random medium with (short) homogeneous extremes where $A$ and $B$ decouple. This gives rise to exact radiation conditions along the outgoing characteristics. Moreover in the time reversal experiments the (long) time reversed signal can be emitted along the incoming characteristic of the domain without interfering with its corresponding outgoing reflected signal. At the homogeneous ends, $x$ is identical to $z$ and it is very easy to recover the physical variables $u$ and $p$.

In a first series of experiments we consider a time-independent medium where only the density is randomly varying $\left(\eta_{i}=\eta_{i i}=0\right)$, with piecewise linear fluctuations $\nu_{i}=\nu_{i i}$. The nodes are equally spaced with increment $h=0.02$. The value taken by the process at node $j h, j \in \mathbb{N}$, is denoted by $X_{j}$. The $\left(X_{j}\right)_{j \in \mathbb{N}}$ is a sequence of independent and identically distributed (i.i.d.) random variables with a uniform distribution over $[-0.5,0.5]$. The initial pulse is the derivative of a Gaussian with unit width and amplitude. We use a square cut-off function $G_{t_{1}}(t)=\mathbf{1}_{\left[0, t_{1}\right]}(t)$ with $t_{1}=100$. Forty different realizations of the process $\nu$ have been simulated, and the refocused pulse shapes for each realization are plotted in Fig. 6. We get excellent statistical stability of the refocused pulse as predicted by the theory. Furthermore, the refocused pulse shape is in agreement with the theoretical formula (convolution of the initial pulse shape with the kernel (42)) as shown by Fig. 8a.

The second series of experiments were devoted to time-dependent media. Once again only the density is randomly varying, but now $\nu_{i} \neq \nu_{i i}$ giving rise to 


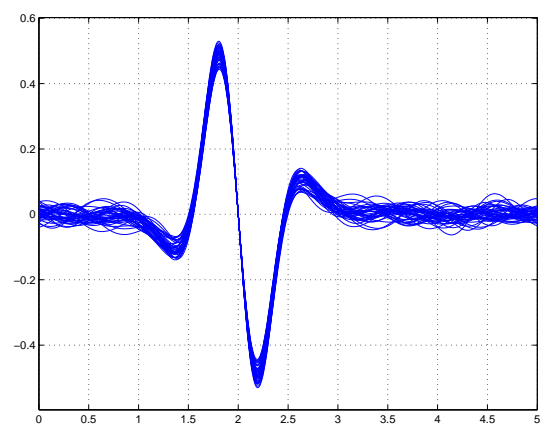

Fig. 6. Refocused pulse shapes for $\alpha_{m}=\alpha_{n}$. The medium is time-independent so that $\delta_{m}=\delta_{n}=1$. We plot 40 experiments performed with different realizations with the same statistical distribution.

(a)

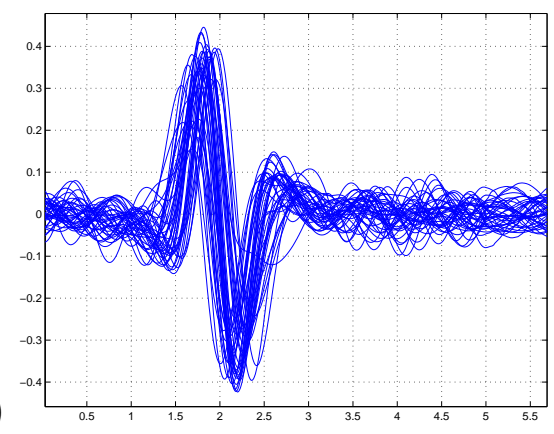

(b)

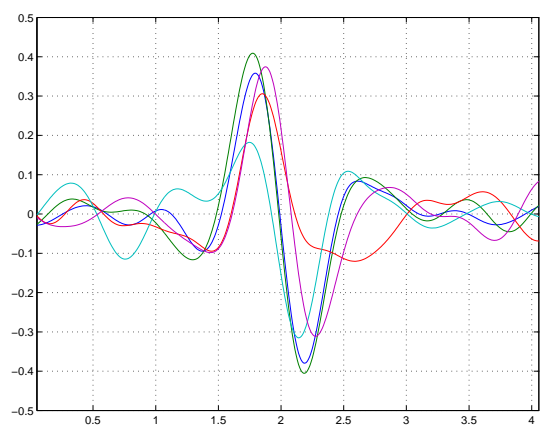

Fig. 7. Refocused pulse shapes for $\alpha_{m}=\alpha_{n}$. Here the fluctuations of the density are time-varying with $\delta_{m}=\delta_{n}=0.75$. The results from 40 experiments are plotted in the left picture. Five individual refocused pulse shapes are taken from this set and plotted in the right picture.

$\delta_{m}=\delta_{n}=0.75$. We can clearly see in Fig. 7 that statistical stability has been lost. However the averaged refocused pulse shape is fully predictable by the theoretical formula (convolution of the original pulse shape with the kernel (43)) as shown by Fig. 8b. By comparing Figs. 8a and 8b we can check that the averaged refocused pulses for the time-independent and the time dependent cases are very similar in shape up to an amplitude reduction factor of order 2 .

We now consider a Goupillaud medium which is widely used in the literature $[3,7]$ and which enables the exact calculations of the reflection and transmission coefficients. In this particular case $\rho(x)$ and $K(x)$ are piecewise constant with jump discontinuities along each layer's interface. These coefficients are such that the travel time across each layer is the same. Accordingly the transmission-reflection matrix $\tilde{M}$ is known exactly and there is no need to discretize the partial differential equation given above [7] since we get directly $\vec{v}^{(n+1)}=\tilde{M} \vec{v}^{(n)}$ where $\vec{v}=(A, B)^{T}$. We have performed a series of experiments with a Goupillaud medium where the travel time across each layer is $h=0.01$. The values of the density and bulk modulus fluctuations $\nu$ and $\eta$ inside each layer are a sequence of i.i.d. random variables, giving rise to a 
(a)

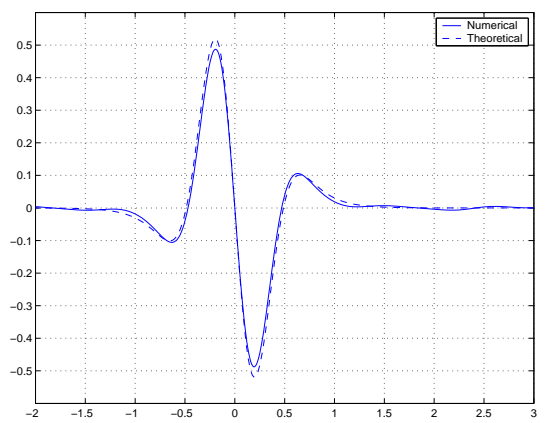

Fig. 8. Comparison of the averaged refocused pulse from the numerical experiments and the mean refocused pulse shape obtained by the asymptotic theory. Picture a: without time perturbation $\delta_{m}=\delta_{n}=1$ (corresponding to Fig. 6). Picture b: with time perturbation $\delta_{m}=\delta_{n}=0.75$ (corresponding to Fig. 7).

(a)

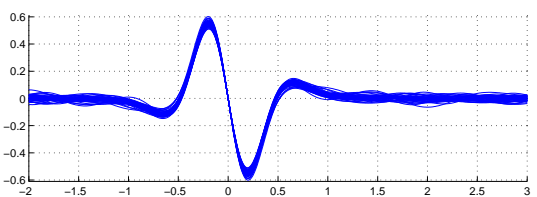

(b)

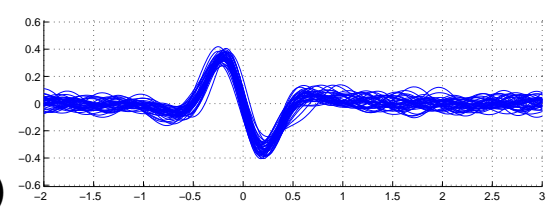

(b)

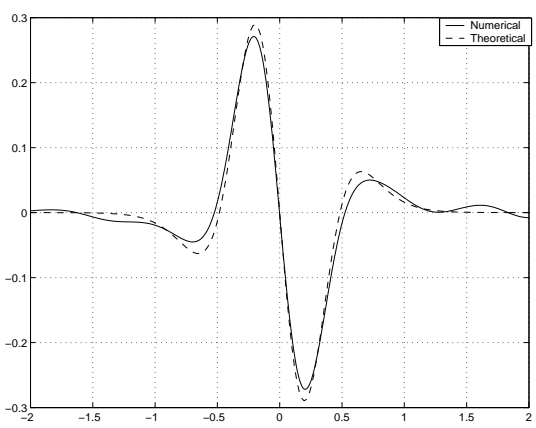

nts 


\section{Conclusion}

We have analyzed the impact of time fluctuations of a random medium on the pulse refocusing during a time-reversal experiment. We have demonstrated that a coherent refocused pulse can still be observed even in the presence of moderate time-perturbations. The recompression of the incoherent reflected signal is very robust in the sense that we always observe a refocused pulse as soon as some correlation degree persists between the two snapshots of the medium. However statistical stability of refocusing is lost in that the refocused pulse shape usually depends on the realizations of the snapshots. Special media such as Goupillaud-type media preserve a strong degree of stabilization, while more general media do not. This is in strong contrast with the results obtained in two recent papers addressing time-reversal in three-dimensional media with weak fluctuations. Statististical stability is numerically observed in the transport regime [5], while statististical stability is demonstrated for the refocused signal in the parabolic white noise limit [6].

Acknowledgements. This work was supported by the ONR grant N0001402-1-0089. J. Garnier was supported by program ACI-NIM-2003-94. A. Nachbin and D. G. Alfaro Vigo were supported by $\mathrm{CNPq} /$ Brazil under grant 300368/96-8.

\section{References}

[1] M. Abramowitz and I. Stegun, Handbook of mathematical functions (Dover, New York, 1965).

[2] D. G. Alfaro Vigo, A. G. Silva Correia, and A. Nachbin, Complete time-reversed refocusing in reflection with an acoustic Lagrangian model, in preparation.

[3] M. Asch, W. Kohler, G. Papanicolaou, M. Postel, and B. White, Frequency content of randomly scattered signals, SIAM Rev. 33 (1991) 519-625.

[4] G. Bal, G. Papanicolaou, and L. Ryzhik, Self-averaging in time reversal for the parabolic wave equation, Stochastics and Dynamics 2 (2002) 507-531.

[5] G. Bal and R. Verastegui, Time reversal in changing environement, to appear in SIAM Mult. Mod. Simul. (2004).

[6] G. Bal and L. Ryzhik, Stability of time-reversed waves in changing media, submitted.

[7] L. Berlyand and R. Burridge, The accuracy of the O'Doherty-Anstey approximation for wave propagating in highly disordered stratified media, Wave Motion 21 (1995) 357-373. 
[8] P. Blomgren, G. Papanicolaou, and H. Zhao, Super-resolution in time-reversal acoustics, J. Acoust. Soc. Am. 111 (2002) 230-248.

[9] R. Burridge, G. Papanicolaou, and B. White, Statistics for pulse reflection from a randomly layered medium, SIAM J. Appl. Math. 47 (1987) 146-168.

[10] J.-F. Clouet and J.-P. Fouque, Spreading of a pulse traveling in random media, Annals of Applied Probability 4 (1994) 1083-1097.

[11] J.-F. Clouet and J.-P. Fouque, A time-reversal method for an acoustical pulse propagating in randomly layered media, Wave Motion 25 (1997) 361-368.

[12] A. Derode, A. Tourin, J. de Rosny, M. Tanter, S. Yon, and M. Fink, Taking advantage of multiple scattering to communicate with time reversal antennas, Phys. Rev. Lett. 90 (2003) 014301.

[13] M. Fink, Time reversed acoustics, Scientific American (November 1999) 91-97.

[14] M. Fink, G. Montaldo, and M. Tanter, Time reversal acoustics in biomedical engineering, Annual Review of Biomedical Engineering 5 (2003) 465-497.

[15] J.-P. Fouque and K. Sølna, Time-reversal aperture enhancement, SIAM Multiscale Modeling and Simulation 1 (2003) 239-259.

[16] H. J. Kushner, Approximation and weak convergence methods for random processes (MIT Press, Cambridge, 1984).

[17] P. Lewicki, R. Burridge, and G. Papanicolaou, Pulse stabilization in a strongly heterogeneous medium, Wave Motion 20 (1994) 177-195.

[18] R. F. O'Doherty and N. A. Anstey, Reflections on amplitudes, Geophysical Prospecting 19 (1971) 430-458.

[19] G. Papanicolaou, Wave propagation in a one-dimensional random medium, SIAM J. Appl. Math. 21 (1971) 13-18.

[20] G. Papanicolaou, Asymptotic analysis of stochastic equations, in MAA Stud. in Math. 18, M. Rosenblatt, ed., Mathematical Association of America, 1978, pp. 111-179.

[21] G. Papanicolaou, L. Ryzhik and K. Sølna, Statistical stability in time reversal, to appear in SIAM J. Appl. Math. (2003).

[22] C. Prada, E. Kerbrat, D. Cassereau, and M. Fink, Time reversal techniques in ultrasonic nondestructive testing of scattering media, Inverse Problems $\mathbf{1 8}$ (2002) 1761-1773.

[23] K. Sølna, Focusing of time-reversed reflections, Waves in Random Media 12 (2003) 365-385.

[24] K. Sølna and G. Papanicolaou, Ray theory for a locally layered medium, Waves in Random Media 10 (2000) 151-198.

[25] A. Tourin, A. Derode, and M. Fink, Sensitivity to perturbations of a timereversed acoustic wave in a multiple scattering medium, Phys. Rev. Lett. 87 (2001) 274301. 